\title{
Modernization of CAPTCHA using Skin Detection Sensors: A Survey
}

\author{
Shrivar \\ Bhuwalka \\ IV year, B.E. \\ Department of \\ CSE \\ BMS College of \\ Engineering
}

\author{
Utsav Seksaria \\ IV year, B.E. \\ Department of \\ CSE \\ BMS College of \\ Engineering
}

\author{
Namratha M. \\ Assistant \\ Professor \\ Department of \\ CSE \\ BMS College of \\ Engineering
}

\author{
Aryan Rai \\ IV year, B.E. \\ Department of \\ CSE \\ BMS College of \\ Engineering
}

\author{
Chaitra Shree \\ IV year, B.E. \\ Department of \\ CSE \\ BMS College of \\ Engineering
}

\begin{abstract}
CAPTCHA is a mechanism to protect the website from malicious programs called bots. The ability of humans to discern distorted characters and to correctly feed them into the computer distinguishes them from bots. With advent in artificial intelligence, the current CAPTCHA mechanisms have developed various flaws. This paper elaborates on the various CAPTCHA mechanisms, their flaws and proposes an alternative technique that uses skin detection to verify human presence.
\end{abstract}

\section{General Terms}

Skin sensing, skin detection, LED based sensors, CAPTCHA techniques, web security, human detection.

\section{Keywords}

CAPTCHA, web security, skin detection, sensors, artificial intelligence, human presence.

\section{INTRODUCTION}

CAPTCHA stands for "Completely Automated Public Turing test to tell Computers and Humans Apart". It is nothing but a box of distorted text or a set of characters written vaguely. The ability of humans to read such characters and the disability of computers to do so, successfully distinguishes them.
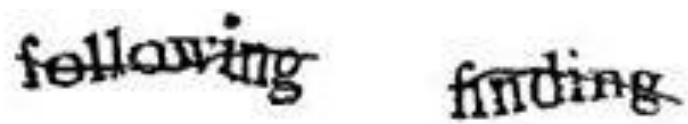

\section{Fig 1 - Text Based CAPTCHA}

Invariant recognition is the identification of a wide range of characters in different shapes and sizes, segmentation is the ability to distinguish one character from the other and parsing refers to the context. A CAPTCHA requires a combination of all the aforementioned to be solved, which humans have and computers treat as NP-hard to be solved in isolation.

With advent of technology and success in artificial intelligence, there have been cases of attack where websites have been spammed and attacked by automated programs, bots. Thus various forms of CAPTCHA have come into the picture. There are image based, video based, audio based and puzzle based CAPTCHAs - all of which have their own technical drawbacks.

In today's fast paced world, CAPTCHAs are proving to be time consuming. They appear everywhere on the Internet, from email registrations and online transactions to just navigating between pages. In Ecommerce and ticketing businesses where each click is crucial, CAPTCHAs have been seen as wastage of time since they become so complicated that even humans read it wrongly. There have been cases where the continuous regeneration of a new CAPTCHA just gives access to the website without even further verifying whether it was a bot or a human that requested the refresh.

It is said that CAPTCHAs are biased, in the sense that specially challenged people face problems in using them. Vision impaired readers suffer problems with text based CAPTCHAs and audio based CAPTCHAs are infamous for the language barrier that they create. Thus, there has been a requirement for an alternative mechanism, which secures the web and the users find convenient to operate on.

A more sophisticated system is one that relies on human senses rather than the reading and identification capabilities of humans. It is better to tap the human sense of skin - a characteristic that every individual would possess and which artificial intelligence can never challenge.

If a sensor, integrated on any standard input device, can successfully detect human skin then there is no need of a challenge response test. This will be a faster method as no separate keying of text or completion of a puzzle will be required.

Skin Detection has been viewed from different perspectives, each of which are relatively new and are finding an increased number of applications.

Firstly, image processing of skin is the most widely used technique. It eliminates the environment around the skin and detects human skin even on big pictures. The technique is used for facial recognition, in photo editing software, to detect obscene images over the Internet and such other applications.

Next is the skin detection by video analytics. This is based on sensing the actual movements of human skin. Such a technique is being used in the wood cutting industry to detect human hands under the woodcutter. Various other applications include identification of human presence in danger zones and industrial equipment.

Finally, skin can also be detected using simple principles of reflection and absorption. Human skin, based on the melanin and blood component will absorb a particular wavelength range and will reflect the rest. Based on the missing bandwidth, it can be detected whether human skin is present or not. This mechanism is the most naïve of all and is gradually finding applications in biometrics.

This Literature Survey Paper intends to shed light on some of the CAPTCHA techniques and their drawbacks. It also 
discusses skin detection as an alternative and explores the existing mechanisms.

\section{LITERATURE SURVEY}

A survey of usability features of CAPTCHA [14] evaluates the currently available techniques of CAPTCHA and tries to determine which of the existing CAPTCHA mechanisms are reliable as well as usable. The authors state that with the increase in reliability, the usability of the CAPTCHA test decreases. They also described the several types of CAPTCHAs, which exist in the world today. CAPTCHAs were classified into various categories like text-based, videobased, and audio based and so on. It was brought to notice that most CAPTCHA mechanisms are not suitable for the use of age groups 5 to 12 and $60+$. Thus stating the age bias of CAPTCHA. To prove their point further, the authors conducted a survey that was taken by a varied group comprising of people of all age groups. The survey required the takers to fill a questionnaire of 30 questions about existing CAPTCHAs. They categorized the speed of a person to solve a CAPTCHA into five categories-Excellent, very good, good, fair and poor. A meager percentage had good speed while none had very good or excellent speed, the majority being either fair or poor. Thus CAPTCHAs currently being used are time consuming. They even asked which a better mode of input was for CAPTCHAs and people preferred the mouse over keyboard as during use of Internet and the advent of touchable devices, people use mouse more than the keyboard. This paper highlighted the distaste of people and the need of a quicker and more convenient mechanism of human identification.

CAPTCHAs are found in a wide variety of applications, from registration of users for a new email id and booking travel tickets to protection of websites in education purposes. CAPTCHAS prevent false registrations by malicious programmers, thus protecting the website from spammers and other harmful content which might retrieve critical information or slow down the website. These malicious programs are bots [13], CAPTCHAS protect the website from these bots.

CAPTCHA is a challenge response test, where the user is shown distorted text or images and he needs to key in the correct letters or chose the correct image based on the thinking capabilities, which a bot would lack. The definition of a CAPTCHA, thus encompasses the properties of a CAPTCHA - it should be easy for human users to understand and pass, easy for a machine to generate and grade and difficult for a software to pass.

Traditional CAPTCHAs are based on the principles of Invariable Identification, Segmentation and Parsing. The identification of letters across various shapes and sizes is called invariable identification; the difference in letters and the individuality of each character from the next is segmentation while parsing refers to the semantic analysis. All three principles are NP-Hard problems in isolation thus difficult for a computer solve. For humans, it is easy to solve all together, thus making it easy to be solved. With advancement in artificial intelligence, combination of aforementioned principles has fallen into the category NP Complete Problems. ReCAPTCHA that uses the OCR technology to distinguish words has replaced traditional CAPTCHA mechanisms. However, a Russian Programmer has cracked the RECAPTCHA with a success rate of $35 \%$.

Ved Praksah Singh and Preet Pal elaborate on the various CAPTCHA mechanisms [12] in place. They explain how each of the mechanisms work. This paper however focuses on the drawbacks of these mechanisms.

Blurred Letters, Multiple fonts and vague designs make textbased CAPTCHAs difficult to read. Users often make mistakes in filling these characters. Optical Character Recognition (OCR), used to generate CAPTCHAS are being used to read text based CAPTCHA. Low vision and blurred images cause problems with image based CAPTCHA. Colorful images are known to create bias against the visually impaired. Audio-based CAPTCHAs are a problem because of various language barriers and similar sounding phonetics. Video-CAPTCHAs have a large size and consume a lot of bandwidth. Also, it is complicated as compared to a simple web based task. Puzzle based CAPTCHAs are time consuming.

Online polling sites that use the above CAPTCHA mechanisms have reported altered results due to attack by bots. Microsoft Live Mail also reported cases of spamming due to the failure of CAPTCHA mechanisms.

There is a patent that talks about a method of verifying the identity of target individuals using near infrared spectrum. [7]

The identity of a target individual can be verified by obtaining target tissue spectral data from the target individual by projecting near-infrared radiation onto the underside of the forearms. Multiple such tissue spectra may be taken from individuals whose identity verification may later be required and stored in a database. By comparing the stored tissue sample against the one that will be acquired at the time of verification one can confirm the identity of the target individual.

The proposed system has a negligible false positive rate but despite the many advantages there are a few concerns. Firstly, even though memory might not be a criterion this technique maintains a huge database that has a heavy toll on memory. It is needless to mention the importance of developing a system that is not so taxing on the memory. Secondly, any failure in connection to the database or corruption of the data in the database will cause the system to fail. Also, it is important to monitor the performance of the system when there are any deformities to the hand whose tissue samples were stored in the database.

There is also a patent that talks about the detection of human body based on its capability to reflect near-infrared bands [1] when exposed to it.

The object under consideration is illuminated by a nearinfrared light source and the reflected bands from the said object are monitored for specific bands using three detectors. The three detectors are used to detect varying reflected bands from the three major skin tones identified in humans, namely, dark, medium and light. The simultaneous detection of a signal of proper reflection values from each of the three detectors confirms the said object as that of a human body.

There are, however, a few drawbacks with this technique. It considers 3 types of skin (light, medium, dark) but it would not be possible to categorize all humans with only such skin tones.

Also it may be possible that certain skin tones are affected by climate or unfortunate illness that do not fall under these categories. Secondly, when working with radiations it is somewhat of a challenge to maintain these bands of radiation within desired limits. It would also be fair to assume that since the proposed system depends on the readings from three 
detectors, the chances of one of them failing and hence the system failing would be higher.

George A. Kilgore and Rand Whillock talk about a method used for skin detection that is based on the IR absorbing and IR reflecting properties [4] of the skin. The skin reflects IR bands in the region of 0.8 to about 1.4 micrometers and absorbs IR bands in the region of 1.4 to about 2.2 micrometers. In the proposed design the illuminator emits 2 IR bands: a first IR band (0.8-1.4) and a second IR band (1.42.2). The detector receives these bands after they come in contact with the skin and the processing unit confirms the skin to be of a humans' on detecting the presence of first IR band and the absence of the second IR band.

The processing unit processes the signal by performing weighted difference and threshold of the outputs from the detectors. If the voltage pulled is higher than the threshold average then its human skin or else it is not. Currently there are no devices that prevent synthetic objects from passing as human skin and hacking biometric systems. But the proposed system could come in handy in these situations and improve the existing security norms.

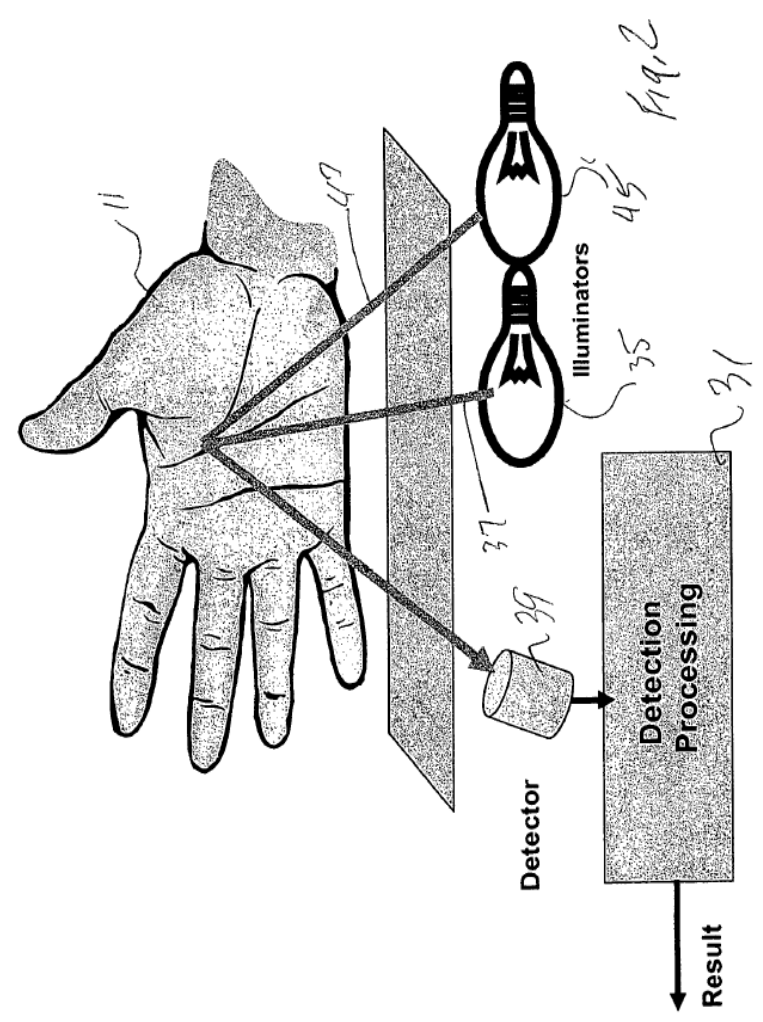

Fig 2 - Skin Detection using 2 IR Bands

A comparative study conducted at the University of Dhaka evaluates the skin detection algorithms and concludes that the main challenges in skin detection are to make the recognition robust to large variations in the appearance of the skin. Skin may vary both in color and texture. Considering the broad spectrum of varied human skin types, these algorithms generally tend to be inefficient and less proficient in skin detection. Also, time taken for detection is high.

Human Skin has a characteristic color and it is a commonly accepted idea driven by logic to design a method based on skin color identification. The problem is there is a lot of variety in human skin colors and textures across the world. For robustness, a huge database needs to be maintained, making the algorithm memory heavy and time consuming.

The inventors thought of using the reflective capabilities of skin that is different from any other similar material. The Infrared Radiation [5] (IR) band of wavelength $1.4 \mu \mathrm{m}$ to 2.2 $\mu \mathrm{m}$ can be completely absorbed by the skin. Thus when incident on skin, the IR detector must not detect any voltage, or at least detect very low voltage. This would correctly identify skin.
Gary E. Determan and David J Wunderlin presented a method of Skin detection using sensors [2]. In this method a LEDemitter emits blue light with a wavelength between 400 to 500 $\mathrm{nm}$. The skin very well reflects blue light within this range. A photodiode or phototransistor records the voltage after reflection of this light from the test material. The recorded voltage is compared to the normalized value (approximately $2.6 \mathrm{~V}$ ). If the value is within the tolerance limits, the material is identified as skin. A set of other articles was also used as target and their values were identified. Skin could thus be correctly identified using this method. An ideal wavelength for the experiment is $468 \mathrm{~nm}$.

The authors also conducted the experiment with other sources of light at varying frequencies. Skin could be detected using other sources of light also, however, distinguishing skin from the other materials was difficult as the recorded voltages were very close to each other. In case of blue light, the value of finger came to be $56 \%$ in isolation from other materials. 


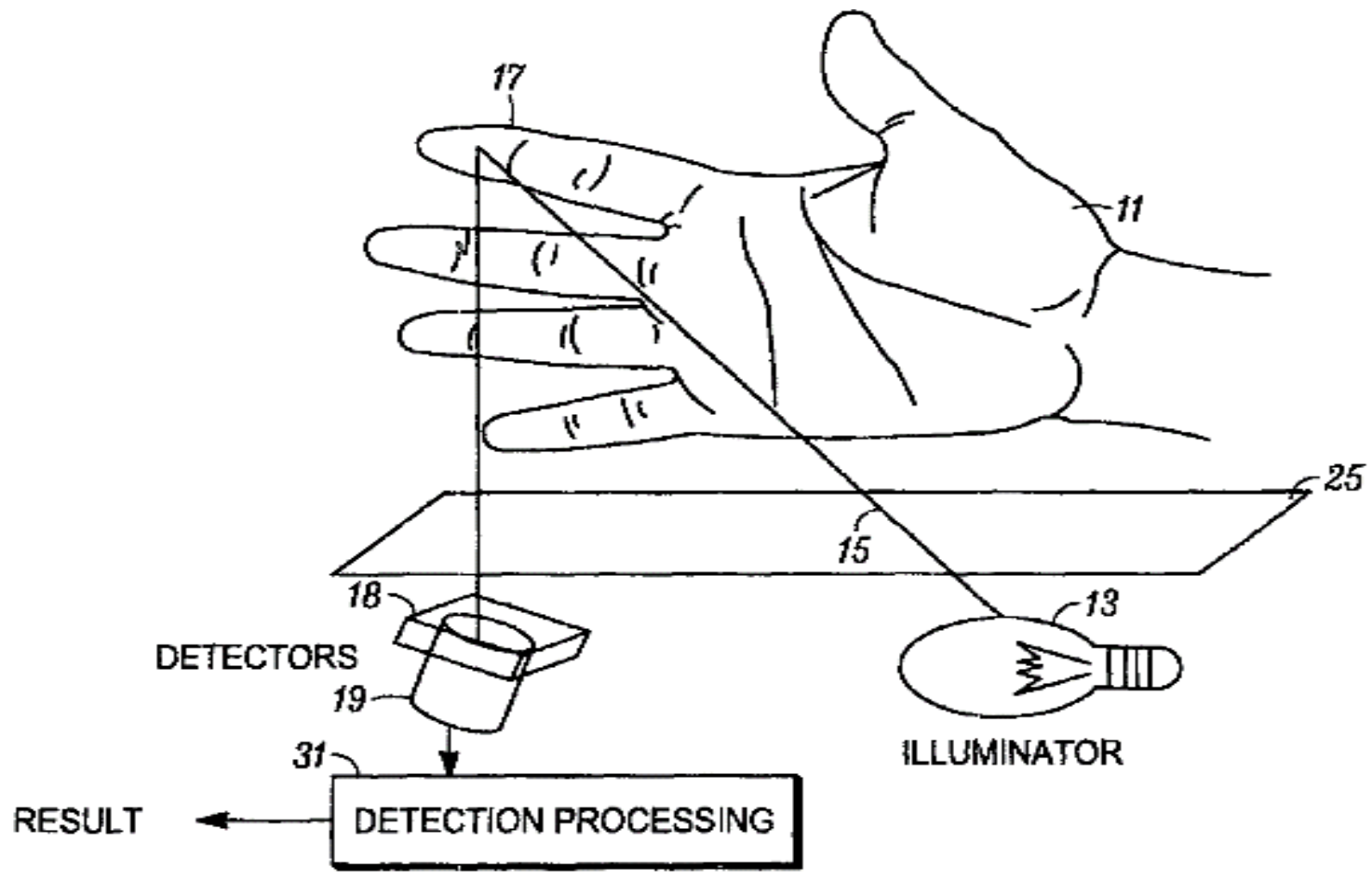

Fig 3 - Skin Detection using Blue Light

Alaa Y Takaa, and Hamid A Jalab [15] say that skin detection is a well-known processing technique. They have proposed techniques that use the color and texture methods, as discussed in the previous papers. These algorithms are reliable because human skin can be detected due to its distinctive components - blood and melanin. Due to the unique color and texture imparted by these components, different ranges of bandwidth will be absorbed and reflected. The challenge is to find out a range of bandwidth as human skin color has its own variations - depending on humans coming from different races of the world. This can however, be generalized using the methods discussed previously.

It was found that the proposed skin detection method achieved a true positive rate of approximately $95.62 \%$ and a false positive rate of $0.89 \%$. The proposed skin detection system, according to the authors, is much better than the state of art skin sensors.

\section{CONCLUSION}

In this paper, a detailed analysis of the CAPTCHA has been performed, highlighting the various social and technical shortcomings of the present methodology. It was seen how various CAPTCHAs have been bypassed unfairly, leading to spammed websites. The requirement for newer technologies has led to thoughts about using human senses rather than human thinking capabilities, to verify human presence.

Skin, the fundamental sense organ, can thus be used to detect human presence. The untapped technology of skin sensing is yet to be fully exploited yet it seems to provide a proper solution to the CAPTCHA problem. The applications and shortcomings of various skin detection techniques have also been discussed in the paper.

A Comparison of all the skin detection techniques mentioned in the paper revealed that the method using reflection of blue light by the skin seems to be highly effective and cost efficient. The only possible drawback seems to be the fact the different skin colors might cause different bandwidths to be reflected, which can be overcome during implementation.

\section{ACKNOWLEDGMENTS}

The work reported in this paper is supported by the college through the TECHNICAL EDUCATION QUALITY IMPROVEMENT PROGRAMME [TEQIP-II] of the MHRD, Government of India.

\section{REFERENCES}

[1] Michael Hacskaylo and Prince Charles CL,"Automatic Human Body Detector", United States Patent, PatentNumber: 4,500,784, Date of Patent: 19 February 2015.

[2] Gary E. Determan and David J. Wunderlin, "Skin Detection Sensor", United States Patent, Patent Number: US 8,098,900 B2, Date of Patent: 17 January 2012.

[3] Phillipe Kahn and Arthur Kinsolving, "Method and apparatus of monitoring the status of a sensor, monitor, or device (SMD)", United States Patent Application Publication, Publication Number: US7849184 B1, Publication Date: 7 December, 2010

[4] George A. Kilgore and P. Rand Whillock, "Skin Detection Sensor", United States Patent, Patent Number: US 7,446,316 B2, Date of Patent: 4 November 2008.

[5] George A. Kilgore, Rand P. Whillock and Gary E. Determan, "Skin Detection Sensor", United States Patent Application Publication, Publication Number: US 2007/0232929 A1, Publication Date: 4 October, 2007.

[6] George A. Kilgore and P. Rand Whillock, "Skin Detection Sensor", United States Patent Application Publication, Publication Number: United States Patent Application Publication, Publication Number: WO2007053450 A1, Publication Date: 10 May 2007. 
[7] Robert K. Rowe, William A. Miller, Nanxiang Ge and Mark Ries Robinson, "Apparatus and method for identification of individuals by near-infrared spectrum", United States Patent Application Publication, Publication Number: US6628809 B1, Publication Date: 30 September, 2003.

[8] George W. McClurg, John F. Carver, Walter G. Scott and Gregory Zyzdryn, "Biometric Imaging System And Method", United States Patent Application Publication, Publication Number: US 2003/0142856 A1, Publication Date: 31 July 2003.

[9] George W. McClurg, John F. Carver, Walter G. Scott and Gregory Zyzdryn, "Biometric Imaging System AndMethod", United States Patent Application Publication, Publication Number: US 2003/0142856 A1, Publication Date: 31 July 2003.

[10] Philip Dean Lapsley, Jonathan Alexander Lee, David Ferrin Pare, Jr. and Ned Hoffman, "Anti-fraud biometricscanner that accurately detects blood flow", United States Patent Application Publication, Publication Number: US 5737439 A, Publication Date: 7 April 1998.

[11] Steiner, H.; Sporrer, S.; Kolb, A. \& Jung, N.: "Design of an Active Multispectral SWIR Camera System for Skin Detection and Face Verification", Journal of Sensors, 2015.

[12] Ved Prakash Singh and Preet Pal, "Survey of Different Types of CAPTCHA", (IJCSIT) International Journal of Computer Science and Information Technologies, Vol. 5 (2), 2014
[13] Baljit Singh Saini and Anju Bala, "A Review of Bot Protection using CAPTCHA for Web Security", IOSR Journal of Computer Engineering (IOSR-JCE) e-ISSN: 2278-0661, p- ISSN: 2278-8727Volume 8, Issue 6(Jan. Feb. 2013), PP 36-42.

[14] Rezvan Pakdel, Norafida Ithnin and Mohammad Hashemi, "CAPTCHA: A Survey of Usability Features", Research Journal of Information Technology 3 (4): 215228, 2011.

[15] Alaa Y. Taqa and Hamid A. Jalab, "Increasing the reliability of skin detectors", Scientific Research andEssays Vol. 5(17), pp. 2480-2490, 4 September 2010.

[16] Jones M.J and J.M. Rehg, "Statistical Color Models with Application to Skin Detection", International Journal of Computer Vision, 46(1): 81-96. DOI: 10.1023/ A: 1013200319198.

[17] Albiol, A.; L. Torres and E.J. Delp, "Optimum color spaces for skin detection", International Conference on Image Processing, October 7-10, 2001, Vol 1, pp 122124. DOI: 10.1109/ICIP.2001.958968.

[18] Martinkauppi, B. and M. Soriano, "Basis Functions of The Color Signals of skin Under Different Illuminants", Third International Conference on Multi-spectral Color Science, June 18-20, 2001. pp 21-24.

[19] Luis von Ben Maurer, Collin McMillen, David Abraham and Manel Blum, "ReCAPTCHA: Human Based Character Recognition via Web Security Measures". 